Revista General de Información y Documentación ISSN: 1132-1873

http://dx.doi.org/10.5209/RGID.60810

\title{
Georreferenciación y publicación web de cartografía antigua en sistemas de información geográficos: requisitos para su evaluación y estudio de caso
}

\author{
Jesús Cascón Katchadourian; Antonio Ruiz Rodríguez²; Adolfo Quesada Román ${ }^{3}$
}

Recibido: 15 de agosto 2017 / Aceptado: 18 de enero de 2018

Resumen. La cartografía con la llegada de las nuevas tecnologías está de moda, el consumo de mapas a gran escala es una realidad gracias a Google Maps y los GPS. Estas tecnologías han hecho llegar su influjo también a la cartografía antigua, a la que le están dando un nuevo aire con la modernización en el uso y tratamiento de los mapas antiguos. Los procesos que hacen esto posible son, entre otros, la digitalización, la georreferenciación y la publicación web. Estos dos últimos procesos se realizan con los Sistemas de Información Geográfica (SIG). El objetivo de este artículo es obtener un listado de requisitos que debe de cumplir un SIG para realizar estos procesos de forma satisfactoria para el usuario, al igual que poner a prueba el software libre QGIS con este listado. La finalidad de esta investigación es ofrecer conocimientos y herramientas válidas para los bibliotecarios, archiveros y otros profesionales de la información que quieran revitalizar sus colecciones cartográficas.

Palabras clave: Cartografía antigua; Georreferenciación; Sistemas de Información Geográfica; QGIS; Evaluación de software.

\section{[en] Georeferencing and web publication of ancient mapping in geographic information systems: requirements for its evaluation and a case of study}

\footnotetext{
Abstract. With the arrival of new technologies the cartography is in fashion, the consumption of large-scale maps is a reality thanks to Google Maps and the GPS. These technologies have also brought their influence to ancient cartography, which is giving a new air with the modernization in the use and treatment of ancient maps. The processes that make this possible are, among others, digitization, georeferencing, and web publishing. These last two processes are carried out with the Geographic Information Systems (GIS). The objective of this article is to obtain a list of requirements that a GIS must fulfill in order to perform these processes satisfactorily for the user, as well as to test the free software QGIS with this listing. The purpose of this research is to provide valid knowledge and tools for librarians, archivists, and other information professionals who want to revitalize their cartographic collections.

1 Departamento de información y comunicación científica. Facultad de Comunicación y Documentación, Universidad de Granada.

E-mail: cascon@ugr.es

2 Departamento de información y comunicación científica. Facultad de Comunicación y Documentación, Universidad de Granada.

E-mail: aangel@ugr.es

3 Escuela de Ciencias Geográficas, Universidad Nacional de Costa Rica.

E-mail: adolfo.quesada@gmail.com
} 
Keywords: Ancient cartography; georeferencing; Geographic Information Systems; QGIS; Software evaluation.

Sumario. 1. Introducción; 2. Metodología; 3. Resultados y discusión; 4. Conclusiones; 5. Referencias bibliográficas.

Cómo citar: Cascón Katchadourian, J.Ruiz Rodríguez, A. Quesada Román, A. (2018) Georreferenciación y publicación web de cartografía antigua en sistemas de información geográficos: requisitos para su evaluación y estudio de caso, en Revista General de Información y Documentación 28 (1), 193-212.

\section{Introducción}

La importancia de la cartografía antigua radica en que son representaciones del entorno del ser humano, tanto ciudades como territorios más amplios, no son sólo meros hechos materiales (Calatrava; Morales, 2005: 14-19). Si sólo fueran imágenes fijas de la evolución de una ciudad o un territorio, la cartografía antigua tendría una gran importancia como fiel reflejo del discurrir del paso del tiempo en un determinado espacio, pero son más que eso, una amplia metáfora de la vida material y mental de los grupos (Fimiani, 1985).

Los mapas no son sólo meros documentos descriptivos y aunque, la importancia que el perfeccionamiento de la técnica tiene es capital para mostrar más fielmente el territorio que quiere mostrar el mapa, se nos antojan fundamentales otros aspectos que a menudo no son estudiados. Por tanto, estos mapas muestran lo que la sociedad del momento demandaba de un mapa, y esta demanda no es una constante, ya que, por ejemplo, el perfeccionamiento técnico, la cuadrícula, la escala, son elementos técnicos que reflejan el pensamiento de una época, la aspiración moderna al control total del espacio. La cartografía antigua, por tanto, no es sólo fuente de información sino en sí misma forma parte activa de la historia.

La importancia de los mapas antiguos también queda reflejada, como veremos a continuación, en que están de moda debido a la modernización de la que han sido objeto en los últimos años. Para este trabajo hablamos de cartografía antigua y no de cartografía histórica, siguiendo a Crespo y Fernández (2011: 371-388) y a la International Cartographic Association (1973), creemos que es la expresión más correcta para referirnos a esa cartografía que en un momento del pasado tuvo vigencia y que el paso de los años la ha convertido en objeto de estudio histórico, pero no en cartografía histórica.

Con la aparición hace 12 años de los servidores de mapas como Google Maps, Bing Maps, OpenStreetMap y otros, se ha producido un auge del consumo de mapas sin precedentes en la historia a nivel de usuario medio (CascónKatchadourian y Ruiz-Rodríguez, 2016: 971). Ello se ha producido por los avances tecnológicos que se han dado en años anteriores en el tratamiento, difusión y manipulación de los mismos, como la restitución por fotogrametría aérea y en la cartografía digitalizada (Cortés-José, 2001: 39-116) los logros conseguidos con los sistemas de posicionamiento global (GPS) y los nuevos procedimientos en la 
composición y redacción cartográficas y en la impresión de mapas (Espiago, 2001: 185-222).

Por último destacar la transformación que supuso, como en todos los ámbitos de la vida, la aparición de Internet y su influencia en la documentación y la cartografía (Jiménez-Pelayo; Bonachera Cano, 2001: 223-266). En la actualidad, gracias a esos avances, han surgido numerosos programas o plataformas digitales de fácil acceso para visualizar, comparar y editar mapas como son OpenLayer (OpenLayer, 2017), Leaflet (Leaflet, 2017), Carto (Carto, 2017) y Maptiler (Cascon-Katchadourian; Ruiz-Rodríguez, 2016: 970-978).

El término georreferenciación es fundamental en este trabajo, según Dávila y Camacho (2012) es un método que permite relacionar información de la posición entre documentos cartográficos de diversa procedencia, existiendo por tanto dos sistemas de coordenadas: el de origen y el de destino. El de origen de la imagen ráster (ESRI, 2013b) digitalizada del mapa antiguo son los valores de fila y columna de los píxeles, no tiene por tanto coordenadas geográficas. Para darle coordenadas geográficas (georreferenciarlo) normalmente se necesita un mapa ya georreferenciado de apoyo o cartografía de referencia e identificar puntos homólogos en ambos sistemas de coordenadas. En un sistema de información geográfico (SIG) el proceso normalmente consiste en pinchar en la cartografía de referencia en un lugar que comparta, sin lugar a dudas, con el mapa sin georeferenciar, posteriormente se pincha en ese mismo lugar en este segundo mapa; de esta forma se le está indicando al programa, que esos dos puntos en ambos mapas son geográficamente el mismo. Lógicamente al tratarse de cartografía antigua hay que buscar lugares que no hayan cambiado en todos estos años: accidentes geográficos, monumentos, calles, u otros. De esta manera se están creando puntos de control, cuanto mayor número de puntos de control creemos, más precisa será la georreferenciación.

La georreferenciación es una importante área de estudio dentro del campo de la cartografía. Si se habla de cartografía antigua, no puede faltar este concepto clave. Se puede dar en cartografía antigua y en cartografía moderna, sin embargo en la cartografía antigua tiene una importancia capital, debido a que ésta no dispone en la mayoría de los casos de coordenadas geográficas en las que situarse, sobre todo si hablamos de planos a gran escala y sin proyecciones. Esta técnica nos permite situar a la cartografía antigua en una situación geográfica determinada y actual, lo que favorecería su comparación con las dinámicas contemporáneas.

Entendemos que este tema es muy relevante y de gran actualidad ya que están apareciendo los medios técnicos para darle a la cartografía antigua que permanece en los depósitos de archivos, bibliotecas y otros centros culturales, otra visión. Unida a la digitalización de estos documentos, la georreferenciación nos permite dar un aire de modernidad a los mapas y nos posibilita visualizarlos desde casa u oficina a través de la web y sin necesidad de desplazarnos, superpuesta sobre mapas tipo Google Maps, Bing Maps u otros. La bibliografía sobre georreferenciación es abundante y reciente (Long et al., 2016), tanto en formato artículo de revista como en monografías de editoriales prestigiosas (Hill, 2009) e incluso de patentes (Witmer et al., 2006). 
La georreferenciación se realiza con un software específico que además de otras muchas funciones, permite este proceso, como son los Sistemas de Información Geográfica. El problema es que con el surgimiento del software libre, que ya ha llegado hasta programas muy complejos como los SIG, nos surge la dificultad de tener que elegir entre uno de los innumerables softwares de este tipo que tenemos a nuestra disposición (QGIS, Gvsig, Grass, Saga, MapInfo u otros) o de los softwares privativos (ArcGIS, Geomedia, Maptiler, Georeferencer u otros). Esta investigación contribuye a tener un conocimiento más profundo de este campo de conocimiento y aportar una herramienta que nos permita evaluar estos softwares en este ámbito tan preciso.

En realidad el término SIG es bastante genérico, dentro de este término hay que incluir programas de muy diversa índole, desde los clásicos SIG de escritorio como serían ArcGis o QGIS, las aplicaciones SIG online como Mapbox o Leaflet o, en lo que a nosotros nos interesa, programas como Maptiler o Georeferencer de Klokantech que "sólo" permiten georreferenciar documentación cartográfica (tanto antigua como no) y convertirla en una capa de mapa interactiva que puede ser mostrada de muy diversas formas a través de Internet. Estos programas, van de más complejos a más sencillos de usar por el usuario novel, según se han ido nombrando.

Es necesario evaluar un SIG en lo relativo a la georreferenciación de cartografía antigua, puesto que son necesarios para poder comparar mapas del pasado y del presente, o de un pasado lejano con otro más cercano, de esta manera se pueden detectar cambios diacrónicos de los espacios geográficos a partir de la cartografía antigua. En la actualidad hay diversas instituciones que están ofreciendo los mapas antiguos georreferenciados (Instituto Geográfico Nacional, 2017a; Instituto Geográfico Nacional, 2017b; Institut National de l'information géographique et forestiére, 2017) y listos para ser comparados e incluso permiten la georreferenciación colaborativa (British Library, 2017; Institut Cartogràfic i Geològic de Catalunya, 2017; National Library of Scotland, 2017; New York Public Library, 2017).

Esta investigación está basada en las experiencias que han tenido los autores con el manejo de cartografía antigua, tanto en la Universidad de Granada, como en la Universidad Nacional de Costa Rica, a través de dos proyectos de investigación: el proyecto de tesis doctoral de Jesús Cascón Katchadourian y su director de tesis Antonio Ángel Ruiz Rodríguez en la Universidad de Granada y la actividad permanente de la Escuela de Ciencias Geográficas de la Universidad Nacional de Costa Rica denominada Mapoteca Virtual (Universidad Nacional de Costa Rica, 2017) (Quesada-Román, 2015: 1-13). Brevemente hay que decir que son dos proyectos con numerosos puntos en común que conllevan la digitalización y publicación en la web de documentación cartográfica, proveniente de otras instituciones (Archivo Nacional de Costa Rica y Archivo Municipal de Granada). Los investigadores se han complementado a través de una estancia de investigación ya que vienen de mundos distintos: la documentación y la geografía; a raíz de la cual se pusieron en común los requisitos que tenía que tener un software SIG y las necesidades operativas que teníamos los investigadores cuando trabajábamos con 
documentación histórica en masa a la hora de georreferenciarla, esta investigación es el fruto de ese trabajo.

El objetivo de esta investigación es dejar para la comunidad científica un listado de ítems para poder evaluar un determinado software SIG en lo que se refiere a la georreferenciación y publicación web de cartografía antigua, este listado y su explicación es a su vez una guía de cómo realizar este proceso de la georreferenciación y qué cuestiones tenemos que tener en cuenta para realizarlo con éxito y de una forma rápida. Además este artículo tiene como objetivo poner a prueba este listado evaluando el programa QGIS y mostrar cómo se comporta este programa en relación a nuestro listado.

\section{Metodología}

\subsection{Listado de ítems}

En primer lugar, se realizó el estudio de qué se entiende por georreferenciación y en qué consiste. Para ello se ha analizado la bibliografía existente sobre el tema para entender cuáles son los pasos fundamentales a realizar en el proceso. La misma es abundante y reciente, incluso sobre georreferenciación de cartografía antigua (Roset; Ramos, 2012) (Dávila; Camacho, 2012).

También se ha realizado un estudio en profundidad de la documentación de apoyo que poseen los principales programas SIG: ArcGIS (ESRI, 2013a), QGIS (QGIS, 2017a), Gvsig (GvSIG, 2015), Grass, Geomedia, y Google Earth tanto de sus propias páginas de tutoriales como de páginas web especializadas en software SIG (ejemplos son www.nosolosig.com o www.mappinggis.com).

En segundo lugar, una vez analizada la bibliografía, se han realizado en esta investigación varias pruebas de georreferenciación en distintos programas SIG (QGIS, Arcgis, Maptiler, Gvsig) y se ha tomado nota exhaustiva de cuáles son las decisiones que tomar en cada paso y qué opciones da cada programa en cada momento.

Como producto de todo lo anterior se ha realizado un listado de aquellos ítems, con los cuales debería de cumplir un software SIG que se precie. Dicho listado se ha puesto a prueba evaluando el SIG de software libre QGIS. La elección del mismo se debió al coste cero del programa y al auge que está teniendo en estos últimos años como alternativa libre a ArcGIS por la poderosa comunidad que tiene detrás de su desarrollo.

Una vez realizada esa primera evaluación, se detectaron los fallos y debilidades del listado como pueden ser: ítems repetitivos que pareciendo distintos acaben evaluando el mismo concepto; ítems que agrupan distintos conceptos que deben ser separados en un nuevo ítem por su importancia individual; ítems que están separados y que se detecta que no son tan importantes como parecía y, o bien se eliminan, o se reagrupan. Por último, se reelaboró el listado y se volvió a evaluar con QGIS para comprobar que se han solucionado las disfunciones del listado previo. 


\subsection{Evaluación de un SIG: QGIS}

En este listado se ha optado por que los ítems estén graduados de 0 a 3, en función de si el programa cumple más o menos con lo que se le pide; no siempre es sí o no. También hay ítems que no tienen gradación posible por lo que, si cumplen con el requisito, obtienen un 3 , y si no, un 0 . Es decir se ha optado por una escala de puntuación mixta, sabiendo que cada sistema de puntuación tiene sus pros y contras. Excepto el primer ítem que es si el sistema georreferencia o no, que es Si o No, todos son cuantitativos. Se ha tenido en cuenta la bibliografía correspondiente para evaluación de sistemas de información, ya que un SIG es un sistema de información.

Se han tenido en cuenta sistemas de evaluación que gradúan la evaluación, como los de Antón y Guallar (2014) o Guallar et al. (2012) donde 0 es inexistencia de cumplimiento del ítem, 1 nivel bajo de cumplimiento del ítem, 2 nivel medio y 3 nivel alto. $\mathrm{O}$ bien los similares pero no exactamente iguales (desaparece el 0 ) de Codina (2000: 22), también 1, 2 y 3, con el detalle de que la escala nominal utilizada por él es: aprobado, notable y excelente. También existen escalas de puntuación mixtas como la de Codina (2006: 4) o el de Antón y Guallar (2014) ya mencionado, donde algunos indicadores pueden ser binarios (si/no que nosotros transformamos en 0/3) y otros graduales $(1,2,3)$. En este estudio los ítems se prestan a estas escalas de puntuación mixtas.

Los ítems se mostrarán ordenados en tres apartados con sus correspondientes tablas: a) Requisitos generales que se valoran en el SIG: se evalúa el programa fuera del módulo de georreferenciación (idiomas, plataformas en las que está disponible, e instalación); b) Ítems propios del módulo de georreferenciación; c) Ítems sobre publicación web de los ficheros georreferenciados.

En esta investigación se ha querido ser inclusivos, de lo cual se deriva que hay un gran número de ítems para evaluar los SIG. Ello hace que haya algunos ítems que tienen una importancia mayor que otros, es decir, un peso mayor en la valoración del programa en cuanto a la georreferenciación. Cuando mostremos los resultados obtenidos por QGIS, habrá un resultado total con todos los ítems y otro resultado sólo con los ítems más importantes.

Esta investigación pretende mostrar un listado para evaluar SIG que finalmente darán un resultado numérico o cuantitativo, por tanto debe estar regido por un control de la subjetividad. Para ello se han tomado una serie de medidas a lo largo del proceso de construcción del listado, dicho proceso consiste en, a) elección de los ítems a evaluar, b) que mide cada ítem, c) gradación de cada ítem entre 0 y 3 o de forma binaria y d) ponderación de los ítems ¿Por qué un ítem se considera más importante que el otro?

En el apartado a) la medida tomada para controlar la subjetividad ha sido el estudio y análisis de lo que hay escrito sobre la georreferenciación, lo que ayuda a elegir qué evaluar y qué no. En el apartado b) la medida tomada para controlar la subjetividad pasa por la realización de una explicación exhaustiva del listado que se propone ítem por ítem, para que no haya duda de qué es lo que se pretende evaluar y así evitar también posibles ambigüedades. En el apartado c) la medida tomada ha sido justificar y comentar profusamente la puntuación obtenida por el 
programa en la evaluación de QGIS donde se prueba el listado a modo de ejemplo. En el apartado d) la medida tomada ha sido la explicación al detalle de por qué se considera un determinado ítem importante. Igualmente ya que los resultados de la evaluación de QGIS se van a dar con todos los ítems y con sólo los más importantes el lector es libre de quedarse con unos resultados o con otros.

\section{Resultados y discusión}

\subsection{Listado de ítems para evaluación de un SIG}

En nuestra opinión la técnica de georreferenciación de un SIG se ha de evaluar según los siguientes parámetros o ítems. Se marcan con un asterisco los ítems más importantes. Para evaluar estos criterios y dar una valoración numérica final a cada programa (en su eventual puesta en práctica), se puntúa cada requisito del 1 al 3. Los primeros 10 ítems evalúan el SIG en general. Del 11 al 21 se evalúa el módulo de georreferenciación. Los tres últimos ítems son los referidos a la publicación web.

A continuación se va a explicar con detalle cada uno de los 24 ítems brevemente reseñados:

1. Un primer ítem denominado "Posibilidad georreferenciación", que es eliminatorio. Es el único cualitativo, pues o georreferencia o no georreferencia y, por tanto, no cuenta para la puntuación final. Sirve básicamente para ofrecer un primer acercamiento de cómo se accede al módulo de georreferenciación, si hay que activarlo, si viene de fábrica o se instala aparte.

2. Software libre o precio bajo. El software o es libre o no lo es. Lo es si los usuarios del mismo tienen las cuatro libertades fundamentales. Es decir, el «software libre» es una cuestión de libertad, no de precio (GNU, 2016). Desde este punto de vista no tendría sentido poner un ítem que relacione el software libre con el precio. Sin embargo nosotros queríamos graduar este ítem; estamos de acuerdo con la GNU pero también entendemos que el precio quitaría la libertad de elegir ese software a las personas sin recursos. Un 3 sería para un software libre gratuito y un 0 para un software excesivamente caro.

3. Facilidad de descarga e instalación. Se evalúa la facilidad para descargar el programa desde la página oficial, la instalación del programa y de otras herramientas de las que haga uso como Microsoft Framework, Python, Java, entre otros. Además se evalúa si el programa viene como un paquete básico que se instala de una vez o se ha de instalar el programa y luego una serie de complementos o módulos. También se evalúa la primera toma de contacto con el software.

*4.Documentación y soporte técnico. En este punto se está evaluando las posibilidades que tiene el usuario de obtener información sobre cómo utilizar el programa. Estamos hablando de información fidedigna y oficial y en menor medida foros no oficiales, videos de youtube, entre otros. Hay numerosos tipos de canales: Guías de uso, listas de correo, foros oficiales o 
no, chat directo con la organización, grupos de usuarios, rastreadores de problemas, soporte comercial, servicio técnico gratuito o de pago, teléfono gratuito o no, email, servicios de consultoría o asistencia técnica, programas de mantenimiento, o tutoriales. Que el programa tenga una buena y clara documentación es fundamental para los que empiezan a manejar un SIG; estos programas han evolucionado mucho en los últimos años pero no hay que llevarse a engaño, un SIG es un programa complejo que trabaja con información compleja, entendiendo por compleja que las tareas que realiza son múltiples y por tanto las decisiones a tomar son múltiples. Para realizarlas con éxito es fundamental por tanto tener una documentación que te ayude con el proceso, valorándose muy positivamente por nuestra parte que esta documentación no sólo explique los “botones” que tiene el módulo georreferenciador sino también que existan videos oficiales que expliquen cómo se hace una georreferenciación sencilla.

5. Multiplataforma. Queremos evaluar en este punto si el programa es instalable y utilizable en distintas plataformas, entendiendo por plataforma el sistema operativo. No se evalúa que un mismo archivo ejecutable se pueda instalar en varios sistemas operativos, sino que los usuarios puedan instalar el programa en varios sistemas operativos aunque sea con distintos archivos ejecutables. Se trata de un punto importante ya que si un programa se limita a una sola plataforma, está dejando fuera a muchos usuarios, además de que es posible que haya usuarios que quieran usar el programa en varias plataformas. Por otro lado, se evalúa negativamente que esté listo el software sólo para un sistema operativo y que se pueda acceder al mismo desde otros sistemas operativos a través de emuladores o soluciones de compromiso no estables. También evaluamos que se pueda usar en teléfonos móviles, aunque hay que ser consciente de que son programas muy potentes y al día de hoy es complicado que lo pueda soportar un móvil, sobre todo como editor, no obstante no se descarta esta posibilidad en el futuro.

6. Varios idiomas: El hecho de que sólo esté disponible en uno o dos idiomas impide el acceso efectivo de muchos usuarios. Se evalúa positivamente tanto la cantidad de idiomas en la que está disponible como que estos idiomas sean mayoritarios. Estamos hablando de la interfaz de usuario del software de escritorio.

*7. Editar y visualizar metadatos. Se trata de un aspecto fundamental en nuestra opinión, que los SIG no tienen del todo resuelto. No es un asunto de poca importancia; los documentalistas y profesionales de la información saben lo importante que es tener correctamente descrita la información, en este caso la información de carácter geográfico. Cuando el usuario posee numerosa información es muy posible que su recuperación sea complicada, para ello es fundamental que el SIG, como sistema de información que es, ofrezca herramientas para describirla correctamente. Por tanto se evalúa positivamente que los metadatos se puedan visualizar, editar, validar, exportar e importar. También que estándares son los que soporta y si se pueden exportar o importar a otras bases de datos. Se analiza si admite también la descripción de los documentos, no sólo de los estándares propios de la geografía, sino también de estándares de otras disciplinas como son la 
biblioteconomía, la archivística, la documentación o más generalistas, por ejemplo de Dublin Core, ISAD (G), ISBD, u otros. Es interesante también que el programa sea capaz de rellenar automáticamente los metadatos técnicos por ejemplo coordenadas, sistemas de coordenadas, y sistemas de referencias.

*8. Cumple con estándares del OCG (Open Geospatial Consortium). El Open Geospatial Consortium (OGC) fue fundado en 1994 para hacer de la información geográfica una parte integral de la infraestructura mundial de información. Los miembros de OGC (usuarios y proveedores de tecnología) desarrollan de forma colaborativa estándares de interfaz y los estándares asociados, así como buenas prácticas, que permiten a los desarrolladores crear sistemas de información que pueden fácilmente intercambiar información geográfica e instrucciones con otros sistemas de información (OSGeo, 2016). Este consorcio es muy activo y está formado por un sinfín de empresas, organizaciones, compañías creadoras de software SIG, estados, o universidades, y ha desarrollado una gran cantidad de estándares (OSGeo, 2014), los más importantes son WMS (Web Map Service), WFS (Web Feature Service), WFS-T (Web Feature Service-Transactional), WCS (Web Coverage Service), WMTS (Web Map Tile Service), TMS (Tile Map Service), WPS (Web Processing Service), SOS (Sensor Observation Service), CSW (Catalogue Service for the Web). Se evaluará si el SIG cumple con estos estándares, bien a través de complementos o del programa básico.

9. Mantenimiento y actualizaciones. En este punto se está evaluando que el programa tenga un equipo detrás que lo supervise y lo actualice con las nuevas técnicas y avances, además de solucionar posibles errores acontecidos. Todo ello se refleja en actualizaciones, versiones nuevas, service packs, parches u otros.

10. Archivos admitidos ráster. Se evalúa en esta sección la cantidad de formatos de archivos ráster que admite el programa, es decir que es capaz de abrir, visualizar y editar. Si abre los fundamentales o los archivos nativos de otros SIG. Estamos por tanto midiendo también la interoperabilidad del software con otros software, es decir, si tiene la capacidad para intercambiar información con otros programas.

*11. Facilidad georreferenciación. Se quieren medir dos cosas: si el módulo de georreferenciación es intuitivo en los primeros contactos con el mismo, ello sin duda redundará en la facilidad para el uso. Sin embargo, es posible que un programa que sea intuitivo para el usuario inexperto se convierta en algo engorroso para el usuario experto. Para el usuario inexperto este punto está en relación con el de la documentación de apoyo anteriormente explicado.

Este punto sería una especie de contenedor donde se incluyen todas las cuestiones que hacen que un software sea más intuitivo y fácil de usar, pequeños detalles que pueden marcar la diferencia. Se valorarían en este punto varías cosas, entre ellas que: sea fácil e intuitivo activar el complemento de la georreferenciación (si es que no está activo con el paquete estándar) y el acceso al mismo desde la interfaz principal del software; permita la georreferenciación por coordenadas escritas 
manualmente o con mapa de referencia (este punto tiene entidad propia como para evaluarse a parte como luego veremos); en el caso que se haga con mapa de referencia estén tanto a la imagen a georreferenciar como la georreferenciada de apoyo en el mismo panel visual, no en distintos y haya que activar y desactivar capas; en esa misma idea que se pueda pinchar en una imagen y que luego al ir a la otra se pueda dar zoom, mover el mapa y luego pinchar y no se desactive la opción de marcar el punto; aunque está valorado por sí mismo en un punto aparte, también hay que valorar aquí, que una vez que hayas puesto dos puntos de control, cuando pones el tercero en el ráster a georreferenciar el programa automáticamente te señale el homólogo en el ráster de apoyo.

*12. Georreferenciación por coordenadas y apoyándose en mapa. De nuevo un punto fundamental; un SIG debe permitir la georreferenciación fundamentalmente a través de dos procedimientos. 1. Meter las coordenadas manualmente; normalmente se introducen con coordenadas X y Y en grados minutos y segundos, o bien con coordenadas proyectadas en metros. 2. Apoyándote en mapa o con cartografía de referencia, es decir con el apoyo de un mapa que sí esté georreferenciado; la técnica consiste en pinchar en el ráster a georreferenciar y en el ráster georreferenciado y establecer puntos de control. Se le están dando básicamente las coordenadas del ráster georreferenciado al ráster a georreferenciar.

*13. Servicio de WMS (Web Map Service). A la hora de hacer una georreferenciación de cartografía histórica es altamente probable que el archivero, bibliotecario o profesional de la información no disponga de cartografía de referencia en su haber para usarla como apoyo en el proceso. Es muy posible que también necesite para hacer la georreferenciación un mapa híbrido que contenga una ortofotografía y una capa con las etiquetas de los nombres de las calles, plazas y monumentos para facilitar el proceso. Descargarse toda esa información no es fácil ni rápido. Es mucho más rápido y cómodo que el SIG venga con un complemento que sirva mapas en streaming, normalmente con el standard del OCG (Open Geospatial Consortium) denominado WMS, aunque también se valorarán otros servicios como WMTS y TMS. Se valora en este ítem que posea esa capacidad y también el número de mapas comerciales que posee, como pueden ser google maps, bing maps, Map Quest, OSM/Stamen, Apple Maps, MapBox, MapQuest, Strava, Tianditu. También se valorará que el programa, aparte de la posibilidad ya comentada, permita acceder a servidores WMS manualmente añadiendo la URL.

*14. Configuración de la transformación del ráster (General). Hemos considerado que la configuración de transformación del ráster es algo sumamente importante. Se analiza con 5 ítems distintos (15, 16, 17, 18, 19), pero también es importante para nosotros que un software tenga una buena puntuación en todos ellos, éste ítem es la media de los siguientes.

*15. a) Tipos de transformaciones del ráster. En este punto queremos evaluar tanto la cantidad como la calidad de los tipos de transformación que tiene el ráster. Pero ¿por qué es importante que tenga varios tipos de transformación? La respuesta es sencilla: cada uno teóricamente sirve más para un tipo de 
documentación y queremos evaluar si el programa es capaz de dar soluciones a los usuarios con distintos tipos de documentación. Por ejemplo la transformación polinómica está optimizada para una precisión global, pero no garantiza la local. El polinomio de orden cero es utilizado normalmente cuando los datos están ya georreferenciados y sólo necesitamos pequeños cambios; el de primer orden desplaza, escala y gira un mapa; el de segundo orden lo dobla o curva; la de spline transforma los puntos de control de origen exactamente en puntos de control de destino (ESRI, 2013a).

*16. b) Métodos disponibles de remuestreo. Cada vez que se modifica la geometría de una imagen ráster, cada vez que hay una deformación elástica vectorial, cambio de la proyección, traslación, giro, entre otros; es muy raro que los centros de celda del ráster de entrada coincidan con los de salida, por lo que hay que realizar un remuestreo (ESRI, 2013a). Cada método de remuestreo es más apropiado para un determinado trabajo. Los tres más usados son: vecino más próximo, interpolación bilineal y convolución cúbica. El primero suele generar un efecto dientes de sierra en las líneas, el segundo difumina los contrastes y el tercero es el mejor para la cartografía escaneada (Dávila y Camacho, 2012). Se valorará que posea los tres y sobre todo el último.

17. c) Output del ráster de salida. Igual que es importante el número de formatos de archivos que el software admite para llevar a cabo la georreferenciación u otras tareas, también es importante el número de formatos de archivo en que el programa es capaz de guardar el ráster georreferenciado. Los ráster georreferenciados tienen que llevar consigo la información de las coordenadas, de la proyección que tienen, bien sea como metadatos dentro del formato Geotiff, o bien como un archivo auxiliar que se sitúa en la misma carpeta que el archivo georreferenciado. Se evalúa en este punto positivamente que el programa admita guardarlos en cuantos más formatos diferentes mejor y a ser posible que esto lo haga en un solo paso.

18. d) Sistema de referencia de coordenadas de destino. Cuando se georreferencia un ráster hay que darle un sistema de referencia de coordenadas de destino; normalmente se ha de poner el sistema de referencia que posee el ráster de apoyo. Se valora que posea el mayor número posible y que el sistema de búsqueda de los mismos sea sencillo y eficiente.

*19. e) Usar 0 para transparencia cuando sea necesario (QGIS, 2017). Que el software posea esta herramienta es fundamental para obtener un buen resultado. Consiste en que cuando se transforma el ráster y se deforma, las partes originales que se contraen dejarían en esa contracción un borde negro desde donde estaba la imagen antes de la transformación hasta el estado actual, una especie de sombra que quedaría como rastro del ráster sin georreferenciar. Con esta opción esa parte queda transparente, por lo que se puede ver la capa de abajo, con lo cual la visualización del ráster georreferenciado es mucho más satisfactoria si lo que queremos es "incrustar" el mapa georreferenciado sobre otro mapa. Se valora por tanto que posea esa herramienta o no; no hay gradación posible, o cero o tres.

*20. Este ítem denominado "Posibilidad de guardar los puntos de control" se refiere a los mismos generados con idea de poder modificarlos a posteriori. 
Expliquemos en qué consiste: esta opción permite ganar tiempo en ciertas ocasiones, por ejemplo cuando se estan haciendo pruebas con un documento a ver qué tipo de transformación, de remuestreo, es el mejor, o bien se está viendo cómo queda mejor la transformación de la imagen con 10 puntos de control, con 20, o incluso más. Si no pudieras guardar los puntos de control, cada vez que haces una prueba te encontrarías que tienes que volver a introducir los mismos con la consiguiente pérdida de tiempo. De nuevo no tendría gradación posible, o permite guardar los puntos para luego usarlos, o no.

*21. Cálculo automático del próximo punto. Consiste en que cuando se está realizando la georreferenciación con un mapa de apoyo ya georreferenciado, una vez se tiene dos puntos de control enlazados entre ambos mapas, cuando se va a introducir el tercero y pinchas en el ráster georreferenciado el programa automáticamente te señala el lugar donde debería ir por triangulación en el ráster a georreferenciar. Lógicamente esta herramienta debe ir acompañada de la posibilidad de modificar ese punto que automáticamente te señala el programa. Te hace ganar mucho tiempo ya que, sobre todo en mapas grandes, te evita alejar el zoom, situarte en el sitio correcto y volver a darle al zoom para pinchar. No tiene gradación ninguna.

*22. Posibilidad publicación web. Se valora en este punto que tenga un módulo o complemento para publicación web de documentos, ya sea documentación vectorial o ráster. De nuevo no tiene gradación, o gestiona el proceso para publicar en la web o no lo hace; con los ítems posteriores ya sí entramos en valoraciones de otro tipo, como los tipos de publicación que permite, o si es fácil o intuitivo.

*23. Facilidad para realizar la publicación web. Intuitivo. En este punto se están evaluando varias cosas: que para realizar la publicación web no se necesite saber código o programación y tener que modificarlo a mano; que no se necesiten configuraciones muy pesadas de los servidores, más propias de empresas grandes.; que se pueda publicar con facilidad tanto datos ráster como vectoriales. El 3 se lo llevaría un software cuyo resultado final fuera una estructura de carpetas listas para subir al servidor con un ftp.

24. Tipos de publicación web. Aunque, como hemos dicho antes, lo ideal para nosotros es que el programa cree la estructura de carpetas para subirlo al servidor. Entendemos que habrá otro tipo de usuarios que necesiten otro tipo de soluciones. Se valora por tanto en este punto que el programa ofrezca diversas formas de publicar en la web, siempre que ofrezcan soluciones diferentes para necesidades de usuarios diferentes.

\subsection{Evaluación de QGIS}

La aplicación del listado de ítems a la evaluación del software QGIS se presenta en una tabla, la cual está dividida en una primera columna de ítems a evaluar, una segunda columna con la puntuación obtenida por QGIS y una tercera gran columna donde se comentan y justifica la puntuación obtenida. A continuación de esta tabla tenemos una segunda más pequeña con los ítems que se consideran más importantes. Esta tabla tiene 2 columnas, ya que se eliminaron los comentarios y justificación. 
A la hora de elegir los ítems que se consideran importantes se han seguido cuestiones puramente técnicas, por eso están casi todos los del módulo de georreferenciación. Se ha entendido que aunque ítems como los idiomas en que está el programa o el sistema operativo para el que está diseñado el SIG son importantes para el usuario, se da por sentado que se ha elegido el SIG teniendo en cuenta esos parámetros y ahora interesa saber quién lo hace técnicamente mejor. A continuación se muestran las tablas que visualizan los resultados que obtiene QGIS con el listado, una tabla por cada módulo (tabla 1, tabla 2, tabla 3), y una última tabla con lo ítems más importantes (tabla 4); se comentarán esos resultados en las conclusiones.

Tabla 1. Módulo requisitos generales.

\begin{tabular}{|c|c|c|}
\hline Item a evaluar & QGIS & Comentarios y justificación \\
\hline $\begin{array}{l}\text {-Posibilidad georreferenciación. } \\
\text { (eliminatorio) }\end{array}$ & SI & $\begin{array}{l}\text { A través de un complemento que viene de fábrica, no } \\
\text { hay que instalarlo aparte, viene con el paquete básico. } \\
\text { Hay que activarlo a través del administrador de } \\
\text { complementos. }\end{array}$ \\
\hline -Software libre o precio bajo. & 3 & Software libre y gratuito. \\
\hline $\begin{array}{l}\text {-Facilidad de descarga e } \\
\text { instalación. }\end{array}$ & 3 & $\begin{array}{l}\text { La descarga e instalación desde la página oficial es } \\
\text { sencilla. La primera toma de contacto para los } \\
\text { principiantes no es compleja. Los distintos } \\
\text { complementos que son los que le dan potencia al } \\
\text { programa son fáciles de instalar, sobre todo si vienen de } \\
\text { fábrica, aunque no estén activados; sin embargo buscar e } \\
\text { instalar complementos que no aparecen en el } \\
\text { administrador es ciertamente complicado. }\end{array}$ \\
\hline $\begin{array}{l}\text {-Documentación y soporte } \\
\text { técnico. }\end{array}$ & 3 & $\begin{array}{l}\text { Hay numerosa documentación tanto oficial como por } \\
\text { parte de los usuarios. La guía de uso (QGIS, 2016a) es } \\
\text { muy completa y, al ser realizada en comunidad, se va } \\
\text { ampliando cada vez más. La única pega es que hay } \\
\text { partes que están traducidas y otras no. El soporte técnico } \\
\text { consiste en listas de correo, foros con StackExchange, } \\
\text { Chat, Grupos de Usuario, rastreador de problemas y } \\
\text { soporte comercial. }\end{array}$ \\
\hline -Multiplataforma & 3 & $\begin{array}{l}\text { Está disponible en Windows, Mac OS X, Linux, } \\
\text { FreeBSD, e incluso hay una experimental para Android: } \\
\text { QField for QGIS. No en Iphone. Obtiene un } 3 \text { porque } \\
\text { está presente en la inmensa mayoría de sistemas } \\
\text { operativos y monta un verdadero SIG para android que } \\
\text { permite crear, editar, visualizar, analizar y publicar } \\
\text { información geoespacial. Muy bien valorado en el Play } \\
\text { Market, con un } 4,2(03 / 08 / 2017) \text {. }\end{array}$ \\
\hline -Varios idiomas. & 3 & $\begin{array}{l}\text { Actualmente está disponible en un buen número de } \\
\text { idiomas, alrededor de } 50 \text {, alrededor porque QGIS está } \\
\text { programado nativamente en inglés, y luego esta } \\
\text { traducido a algunos idiomas al } 100 \% \text { y a otros en un } \\
\text { porcentaje menor. Hay } 10 \text { idiomas traducidos al } 100 \% \text {, } \\
24 \text { de } 50 \text { están traducidos a más del } 90 \% \text {...En QGS } \\
\text { Desktop se puede ver el porcentaje en Ayuda-->Acerca } \\
\text { de-->traductores. }\end{array}$ \\
\hline
\end{tabular}




\begin{tabular}{|l|l|l|}
\hline -Editar y visualizar metadatos. & 1 & $\begin{array}{l}\text { Existe un complemento denominado metatool que se } \\
\text { supone funciona con QGIS, no viene de fábrica y no es } \\
\text { fácil de encontrar. Tras hacerlo QGIS no lo carga } \\
\text { correctamente, lo cual le ocurre a numerosas personas } \\
\text { según se aprecia en los foros. Se puede solucionar } \\
\text { modificando una línea con Python. No es serio. La } \\
\text { versión de fábrica sí posee algunos metadatos que se } \\
\text { pueden visualizar y editar; se accede dándole al botón } \\
\text { derecho en la capa, propiedades-->metadatos. Pero son } \\
\text { escasos: Título, resumen, lista de palabras claves, URL } \\
\text { de datos, atribución (título y URL), URL de Metadatos } \\
\text { (de dos tipos de estándar: FGDC y TC211 y en dos } \\
\text { formatos: TXT y XML), URL de Leyenda. }\end{array}$ \\
\hline $\begin{array}{l}\text {-Cumple con estándares del } \\
\text { OCG }\end{array}$ & 3 & $\begin{array}{l}\text { De los 9 estándares QGIS cumple con 7. Con los otros } \\
\text { dos (TMS y SOS), lo hace a través de plugins o } \\
\text { complementos. }\end{array}$ \\
\hline -Mantenimiento y & 3 & $\begin{array}{l}\text { Se actualiza muy frecuentemente solucionando errores y } \\
\text { otorgando nuevas herramientas. Cada 2 o 3 meses sale } \\
\text { una nueva versión. }\end{array}$ \\
\hline actualizaciones. & 3 & $\begin{array}{l}\text { Admite infinidad de archivos ráster; si le damos a abrir } \\
\text { capa ráster en QGIS vemos que hay 72 categorías de } \\
\text { archivos que abre, algunas de ellas como el manejador } \\
\text { de archivos GDAL abre unos 10 tipos de archivos } \\
\text { distintos. }\end{array}$ \\
\hline -Archivos admitidos ráster. & $\mathbf{2 5}$ & \\
\hline TOTAL PUNTUACIÓN &
\end{tabular}

Tabla 2. Módulo de georreferenciación.

\begin{tabular}{|l|l|l|}
\hline Item a evaluar & Qgis & \multicolumn{1}{|c|}{ Comentarios y justificación } \\
\hline -Facilidad georreferenciación. & $\begin{array}{l}\text { El proceso es intuitivo teniendo en cuenta que para hacer una } \\
\text { georreferenciación necesitamos darle al ráster un SRC (Sistema } \\
\text { de Referencia de coordenadas), un tipo de transformación, } \\
\text { método de remuestreo, compresión, y un sistema de referencia } \\
\text { espacial de destino. La documentación del programa explica el } \\
\text { Intuitivo. }\end{array} \quad \begin{array}{l}\text { proceso paso a paso. Para hacer la georreferenciación se } \\
\text { necesita en QGIS activar el complemento georreferenciador; } \\
\text { esto se hace pinchando en complementos, administrar e instalar } \\
\text { complementos y activar georreferenciador GDAL. Para abrir } \\
\text { este complemento hay que pinchar en Ráster-- } \\
\text { lGeorreferenciador, entra dentro de la lógica ya que la } \\
\text { georreferenciación es para los ficheros ráster. Permite la } \\
\text { georreferenciación por coordenadas o con mapa de referencia, } \\
\text { al que ellos llaman a partir del lienzo del mapa. Ofrece dos } \\
\text { opciones: 1. Que estén ambas imágenes en el mismo panel } \\
\text { visual. Dentro de esta opción puedes pinchar en el ráster a } \\
\text { georreferenciar y luego hacer zoom en el ráster } \\
\text { georreferenciado de apoyo y posteriormente pinchar. 2. Que no } \\
\text { estén ambas imágenes en el mismo panel visual. Obtiene un 2 } \\
\text { porque el software no te predice el punto automáticamente } \\
\text { como veremos más adelante en otro ítem. }\end{array}$ \\
\hline
\end{tabular}




\begin{tabular}{|c|c|c|}
\hline $\begin{array}{l}\text { Georreferenciación por } \\
\text { coordenadas y apoyándose en } \\
\text { mapa. }\end{array}$ & 3 & $\begin{array}{l}\text { Permite meter las coordenadas a mano si las conocemos o usar } \\
\text { una cartografía de referencia poniendo los puntos de control } \\
\text { pinchando en el ráster a georreferenciar y en el de referencia. } \\
\text { Cuando se pincha en el ráster a georreferenciar, } \\
\text { automáticamente te sale una ventana denominada "Introducir } \\
\text { coordenadas de mapa", ahí es donde te da las dos opciones, } \\
\text { escribirlas a mano o a partir del lienzo del mapa. También se } \\
\text { pueden añadir los puntos guardados con anterioridad con un } \\
\text { archivo .points. }\end{array}$ \\
\hline $\begin{array}{l}\text {-Servicio de WMS: tipos de } \\
\text { mapas disponibles. }\end{array}$ & 2 & $\begin{array}{l}\text { A través del complemento Open Layers Plugin (QGIS, 2016b) } \\
\text { se tiene Open Street Maps, Google Maps, Bing Maps, Map } \\
\text { Quest, OSM/Stamen y Apple Maps. Muy completo y rápido } \\
\text { más que por ejemplo ArcGIS (Hasta la actualización de } \\
\text { ArcBruTile). Sin embargo a veces es inestable cuando se } \\
\text { produce una actualización. Se observa que en ArcGIS por } \\
\text { ejemplo hay más zoom; es importante para la precisión, de ahí } \\
\text { el 2. Se valora positivamente que Open Layers Overview te } \\
\text { permite, una vez que has centrado la capa de Google Maps, por } \\
\text { ejemplo, en la zona que te interesa, puedes añadir otra de Bing } \\
\text { que se añade automáticamente en esas coordenadas, de tal } \\
\text { manera que acelera tremendamente el proceso puesto que no } \\
\text { tienes que ir haciendo el zoom hasta la zona propuesta. } \\
\text { También se pueden añadir esas capas manualmente, } \\
\text { poniéndoles título y la url del servidor. }\end{array}$ \\
\hline $\begin{array}{l}\text {-Configuración de la } \\
\text { transformación del ráster } \\
\text { (General) }\end{array}$ & 3 & $\begin{array}{l}\text { La media de todos los procesos que se describen abajo en QGIS } \\
\text { es un } 3 \text {. En los } 5 \text { procesos obtiene una puntuación de } 14 \text { sobre } \\
15 \text {. }\end{array}$ \\
\hline $\begin{array}{l}\text {-a) Tipos de transformaciones } \\
\text { del ráster. }\end{array}$ & 3 & $\begin{array}{l}\text { Tiene varios tipos de transformación, muy completo, cada tipo } \\
\text { de transformación es más apropiado para cada tipo de imagen. } \\
\text { Este tiene: Lineal, Helmert, Polinomial 1, 2, 3, Thin Plate } \\
\text { Spline y Proyectivo. }\end{array}$ \\
\hline $\begin{array}{l}\text {-b) Métodos disponibles de } \\
\text { remuestreo. }\end{array}$ & 3 & $\begin{array}{l}\text { Métodos de remuestreo disponibles: Vecino más próximo, } \\
\text { lineal, cúbica, spline cúbica y Lanczos. Como se observa, tiene } \\
\text { los tres fundamentales, entre ellos el más importante que es el } \\
\text { de convolución cúbica. Además tiene dos tipos más. }\end{array}$ \\
\hline -c) Output del raster de salida. & 2 & $\begin{array}{l}\text { En realidad, QGIS, igual que admite } 72 \text { tipos de archivos } \\
\text { distintos, también admite } 72 \text { tipos de output, por lo que } \\
\text { obtendría un } 3 \text {. Sin embargo se le asigna un } 2 \text { porque eso lo } \\
\text { hace en dos pasos; el complemento de georreferenciación sólo } \\
\text { permite guardarlo como geotiff lo cual ocupa bastante y luego } \\
\text { pinchando en ráster } \rightarrow \text { conversión } \rightarrow \text { traducir lo puedes pasar el } \\
\text { geotiff a los } 72 \text { tipos de output. }\end{array}$ \\
\hline $\begin{array}{l}\text {-d) Sistema de coordenadas de } \\
\text { referencia de destino. }\end{array}$ & 3 & $\begin{array}{l}\text { Tiene infinidad de sistemas de coordenadas y están todos los } \\
\text { importantes. Los sistemas de búsqueda son adecuados y } \\
\text { eficientes. Consisten en una búsqueda por texto en un recuadro } \\
\text { donde pone filtrar, adecuado para los que saben el nombre del } \\
\text { sistema de coordenadas, y el código EPSG. También tiene un } \\
\text { listado donde aparecen todos los sistemas de referencia dividido } \\
\text { en carpetas que cuando se pinchan se despliegan (browsing). El } \\
\text { primer nivel estaría dividido entre: Sistemas de coordenadas } \\
\text { geográficas; Sistemas de coordenadas proyectadas; o sistemas } \\
\text { de coordenadas definidos por el usuario (ya que permite que se } \\
\text { le introduzca manualmente un sistema de coordenadas). Los } \\
\text { sistemas de coordenadas geográficas, están ordenados } \\
\text { alfabéticamente. Los sistemas de coordenadas proyectadas, }\end{array}$ \\
\hline
\end{tabular}




\begin{tabular}{|l|l|l|l|}
\hline \multicolumn{1}{|l|}{} & & $\begin{array}{l}\text { están ordenados en carpetas alfabéticamente (es decir, tiene un } \\
\text { segundo nivel de ordenación). Es interesante también que el } \\
\text { sistema guarde y almacena los sistemas de referencia de } \\
\text { coordenadas usados recientemente. }\end{array}$ \\
\hline $\begin{array}{l}\text {-e) Usar 0 para transparencia } \\
\text { cuando sea necesario }\end{array}$ & 3 & $\begin{array}{l}\text { En QGIS, cuando se quiere georreferenciar una imagen, hay } \\
\text { que configurar la transformación obligatoriamente. En la } \\
\text { pantalla de configuración de la transformación, abajo del todo, } \\
\text { hay una casilla de verificación, que por defecto viene marcada, } \\
\text { denominada "Usar 0 para transparencia cuando sea necesario" } \\
\text { que es la que permite "borrar" la sombra de la deformación del } \\
\text { ráster. }\end{array}$ \\
\hline $\begin{array}{l}\text {-Posibilidad de guardar los } \\
\text { puntos de control. }\end{array}$ & 3 & $\begin{array}{l}\text { En QGIS se pueden guardar los puntos de control. En el } \\
\text { complemento georreferenciador, se guarda dándole a Archivo-- } \\
\text { >guardar puntos PCT como...Una vez hecho esto te permite } \\
\text { guardar los puntos como Archivo GCP (*.points). }\end{array}$ \\
\hline $\begin{array}{l}\text {-Calculo automático del } \\
\text { próximo punto. }\end{array}$ & 0 & No hace el cálculo automático del próximo punto. \\
\hline TOTAL PUNTUACIÓN & $\mathbf{2 7}$ & \multicolumn{2}{|l}{} \\
\hline
\end{tabular}

Tabla 3. Módulo publicación web.

\begin{tabular}{|l|c|l|}
\hline \multicolumn{1}{|c|}{ Item a evaluar } & QGIS & \multicolumn{1}{|c|}{ Comentarios y justificación } \\
\hline -Posibilidad publicación web & 3 & Sí, permite la publicación web de varias maneras. \\
\hline $\begin{array}{l}\text {-Facilidad para realizar la } \\
\text { publicación web. Intuitivo. }\end{array}$ & 1 & $\begin{array}{l}\text { En QGIS, como otros SIG de escritorio, es medianamente fácil } \\
\text { publicar mapas con vectores. Al hablar de ráster, la cosa se } \\
\text { complica bastante, tanto por capacidad de almacenamiento } \\
\text { como por arreglos que hay que ir haciendo. Se entiende que el } \\
\text { público al que va dirigido este artículo no tiene por qué saber } \\
\text { código o programación que incluyen algunas de las opciones de } \\
\text { publicación web de QGIS. }\end{array}$ \\
\hline -Tipos de publicación web. & 2 & $\begin{array}{l}\text { Tiene bastantes tipos de publicación web, como QGIS Server } \\
\text { (bastante complicado) (QGIS, 2017b), QGIS Cloud no permite } \\
\text { raster (QGIS Cloud, 2017), y varios complementos del } \\
\text { programa que te permiten publicar como si fuera leaflet } \\
\text { (Leaflet, 2017), como QGIS2leaf, open layer u open geo, lo que } \\
\text { hacen es conectar varias herramientas de software libre, con } \\
\text { límites de almacenamiento o que directamente son sólo para } \\
\text { información vectorial. Se le asigna un 2 porque da muchas } \\
\text { opciones para usuarios distintos, pero para publicar ráster es } \\
\text { ciertamente complicado. Interesante este artículo de } \\
\text { MappingGIS (2017) }\end{array}$ \\
\hline TOTAL PUNTUACION & $\mathbf{6}$ & \begin{tabular}{l} 
\\
\hline
\end{tabular} \\
\hline
\end{tabular}


Tabla 4. Selección de ítems más importantes

\begin{tabular}{|l|c|}
\hline \multicolumn{1}{|c|}{ Item a evaluar } & Qgis \\
\hline -Documentación y soporte técnico. & 3 \\
\hline -Editar y visualizar metadatos. & 1 \\
\hline -Cumple con estándares del OCG. & 3 \\
\hline -Facilidad georreferenciación. Intuitivo. & 2 \\
\hline -Georreferenciación por coordenadas y apoyándose en mapa. & 3 \\
\hline -Servicio de WMS: tipos de mapas disponibles. & 2 \\
\hline -Configuración de la transformación del ráster (General): & 3 \\
\hline -a) Tipos de transformaciones del ráster. & 3 \\
\hline -b) Métodos disponibles de remuestreo. & 3 \\
\hline -e) Usar 0 para transparencia cuando sea necesario. & 3 \\
\hline -Posibilidad de guardar los puntos de control. & 3 \\
\hline -Calculo automático del próximo punto. & 0 \\
\hline -Posibilidad publicación web. & 3 \\
\hline -Facilidad para realizar la publicación web. Intuitivo. & 1 \\
\hline Total & 33 \\
\hline
\end{tabular}

Fuente: Elaboración propia.

QGIS obtiene 56 puntos de los 69 posibles $(23 \times 3=69)$ es un programa con una gran valoración por parte de nuestro listado; la media de la puntuación por ítem sería de un 2,434, una puntuación bastante alta. Igualmente en los ítems más técnicos e importantes obtiene 33 puntos de los 45 puntos posibles $(15 \times 3=45)$, la media de puntuación por ítem sería de un 2,2, ligeramente por debajo de la evaluación general, pero de nuevo una buena puntuación.

Al mostrar los resultados por tablas, una por cada módulo y hacer la sumatoria por cada una de las tablas, se pueden observar los resultados de cada uno de los módulos. El de características generales obtiene una puntuación de 25 sobre 27, el módulo de georreferenciación obtiene una puntuación de 27 sobre 33 y el modo de publicación web una puntuación de 6 sobre 9.

\section{Conclusiones}

La principal contribución de este estudio es el listado con los principales ítems que hay que tener en cuenta para evaluar la georreferenciación de documentación por parte de un SIG, además de la evaluación de QGIS según este punto de vista.

En los resultados de este artículo se han fijado los requisitos que deben cumplir estos software con un listado de ítem profusamente explicado. El listado por otro lado es a la vez producto y resultado palpable de la sistematización de estas observaciones. Por último este listado es inclusivo, con un gran número de ítems agrupados por categorías, que servirá para evaluar tanto la georreferenciación de cartografía antigua, como la georreferenciación de otra documentación y a modo muy general los ítems de características generales pueden servir como evaluación de un software SIG a nivel global. Hasta el momento no se ha encontrado en la literatura científica ningún instrumento que permita evaluar estos softwares en este apartado. 
Por otro lado, de este estudio se pueden sacar muchas conclusiones por parte de los usuarios que serían imposibles de plasmar aquí, es decir, este listado y su explicación pueden servir como guía al usuario del mismo y luego ellos evaluar los softwares con los ítems que ellos decidan. Con este trabajo se ha ofrecido el listado completo y posteriormente se ha reflejado cuáles son los ítems que tienen más importancia técnicamente. El listado por tanto es ampliable y reducible por el usuario, se pueden escoger los ítems que tienen que ver sólo con el módulo de georreferenciación, con el de publicación, y adaptar el listado a un caso concreto.

En cuanto al otro resultado del estudio, la evaluación de QGIS con el listado, se puede concluir que QGIS es un gran SIG a nivel global pero que pierde un poco de su fortaleza en el módulo de georreferenciación y que tiene pendiente una mejora en cuanto a la publicación web. Este estudio es muy relevante para la comunidad que hay detrás del desarrollo de QGIS que puede sacar recomendaciones de mejora de este estudio y mejorar el programa en el futuro.

En cuanto a las líneas futuras de investigación este trabajo es un primer paso necesario, que implicaría un segundo paso, que nos llevaría a analizar con este listado varios SIG y sacar conclusiones de cuál es el mejor evaluado, es decir, hacer una comparación entre ellos. También es una posible línea de investigación profundizar en la descripción del proceso de georreferenciar documentación histórica así cómo profundizar en el proceso de pasar la cartografía histórica a la web paso a paso con el uso de los software pertinentes.

\section{Referencias bibliográficas}

Anton, L.; Guallar, J. (2014). Análisis de los archivos audiovisuales en internet de las televisiones autonómicas españolas. Revista Española de Documentación Científica, 37 (1): e033.

British Library (2017). Online Gallery. Georeference home. http://www.bl.uk/ georeferencer/[Consulta: 21/07/2017]

Calatrava, J.; Ruiz, M. (2005). Los planos de Granada 1500-1909: cartografía urbana e imagen de la ciudad (Vol. 26). Granada: Diputación Provincial de Granada.

Carto (2017). Carto. Unlock the potential of your location data. https://carto.com/

[Consulta: 21/07/2017].

Cascón-Katchadourian, J.; Ruiz-Rodríguez, A. Á. (2016). Descripción y valoración del software MapTiler: del mapa escaneado a la capa interactiva publicada en la Web. El profesional de la información, 25(6), 970-978.

Codina, L. (2000). Evaluación de recursos digitales en línea: conceptos, indicadores y métodos. Revista española de documentación científica, 23 (1), 9-44.

Codina, L. (2006). Metodología de análisis y evaluación de recursos digitales en línea. Barcelona: UPF, Área de Biblioteconomía y Documentación, Departamento de Periodismo y Comunicación Audiovisual. www.lluiscodina.com/wp-content/uploads/ metodov6.doc_[Consulta: 21/07/2017]

Cortés, J. (2001). El documento cartográfico, en Jiménez Pelayo, J. y Monteagudo LópezMenchero, J. (Eds.) La documentación cartográfica: Tratamiento, gestión y uso. Huelva: Universidad de Huelva, 39-113.

Crespo, A.; Fernández, A. (2011). ¿Cartografía antigua o Cartografía histórica? Estudios Geográficos, 72(271), 371-388. 
Dávila, F. J; Camacho, E. (2012). Georreferenciación de documentos cartográficos para la gestión de archivos y cartotecas: "propuesta metodológica". Revista Catalana de Geografía, 17(46),

Espiago, J. (2001). Documentación cartográfica digital, en Jiménez Pelayo, J. y Monteagudo López-Menchero, J. (Eds.) La documentación cartográfica: Tratamiento, gestión y uso. Huelva: Universidad de Huelva, pp. 183-220.

ESRI (2013a). Principios básicos de georreferenciación de un dataset ráster. http://resources.arcgis.com/es/help/main/10.1/index.html\#/na/009t000000mn000000/

[Consulta: 21/07/2017].

ESRI (2013b). ¿Qué son los datos ráster? http://resources.arcgis.com/es/help/main/10.1/ index.html\#/na/009t00000002000000/ [Consulta: 21/07/2017].

Fimiani, M. (1985). "Cartografie", en Donatella Mazzoleni (a cura di). La città e l'immaginario. Roma: Officina Edizioni, p. 227.

GNU (2016) ¿Qué es el software libre? www.gnu.org/philosophy/free-sw.es.html [Consulta: 21/07/2017].

Guallar, J.; Abadal, E.; Codina, L. (2012). Sistema de análisis de hemerotecas de prensa digital. Trípodos, 31, 37-64.

GvSIG. (2015). Manual de Usuario. En QGIS (Ed.), http://downloads.gvsig.org/download/ gvsig-desktop/dists/2.2.0/docs/gvsig-2.2.0-doc-1.0.0-es.pdf_[Consulta: 21/07/2017].

Hill, Linda L. (2009). Georeferencing: The geographic associations of information. Mit Press.

International Cartographic Association. (1973). Multilingual Dictionary of Technical Terms in Cartography. Dictionnaire Multilingue de Termes Techniques Cartographiqes: Diccionario Multilingüe de Términos Técnicos Cartográficos; F. Steiner. Wiesbaden: International Cartographic Association

Instituto Geográfico Nacional (2017a). Fondos Cartográficos del Instituto Geográfico Nacional. España. Siglos XVI-XIX. www.ign.es/fondoscartograficos/_[Consulta: 21/07/2017]

Instituto Geográfico Nacional (2017b). Comparador de Mapas. http://www.ign.es/web/ mapasantiguos/\#map=5/-1669792.36/4163881.14/0_[Consulta: 21/07/2017]

Institut Cartogràfic i Geològic de Catalunya (2017). Cartoteca Digital. Georeferenciacio. http://cartotecadigital.icgc.cat/cdm/Georeferenciacio/lang/es [Consulta: 21/07/2017]

Institut National de l'Information Géographique et Forestiére (2017). Remonter le temps. https://remonterletemps.ign.fr/ [Consulta: 21/07/2017]

Jiménez-Pelayo, J.; Bonachera-Cano, F.-J. (2001). Recursos de información cartográfica en internet”, en: Jiménez-Pelayo, J.; Monteagudo-López-Menchero, J. (Eds). La documentación cartográfica: Tratamiento, gestión y uso. Huelva: Universidad de Huelva, pp. 221-262.

Leaflet (2017). Leaflet. http://leafletjs.com/[Consulta: 21/07/2017]

Long, T.i; Jiao, W.; He, G.; Zhang, Z. (2016). A Fast and Reliable Matching Method for Automated Georeferencing of Remotely-Sensed Imagery. Remote Sensing, 8(1), 56. www.mdpi.com/2072-4292/8/1/56/htm. [Consulta: 21/07/2017].

MappingGIS (2017). Cómo publicar mapas online con QGIS Cloud. http://mappinggis.com/2012/11/como-publicar-mapas-con-QGIS/ [Consulta: 21/07/2017]

National Library of Scotland (2017). NLS Map Georeferencer home page - online map georeferencing pilot programme. http://maps.nls.uk/projects/georeferencer/ [Consulta: 21/07/2017]

New York Public Library (2017). NYPL Map Warper. http://maps.nypl.org/warper/ [Consulta: 21/07/2017]

OpenLayers (2017). OpenLayers. A high-perfomance, feature-packed library for all your mapping needs. https://openlayers.org/[Consulta: 21/07/2017]

QGIS (2016a). Guía de usuario de QGIS. http://docs.QGIS.org/2.8/es/docs/user_manual/

[consulta: 21 de Julio 2017] 
QGIS (2016b). OpenLayers Plugin. https://plugins.QGIS.org/plugins/openlayers_plugin/ [Consulta: 21/07/2017]

QGIS (2017). Complemento georreferenciador. http://docs.qgis.org/2.2/es/docs/user _manual/plugins/plugins_georeferencer.html_[Consulta: 21/07/2017]

QGIS (2017b). QGIS como Servidor de Datos OCG. http://docs.qgis.org/2.2/es/docs/ user_manual/ working_with_ogc/ogc_server_support.html_[Consulta: 21/07/2017]

QGIS Cloud (2017). QGIS Cloud Hosting. https://QGIScloud.com/_[Consulta: 21/07/2017]

OSGeo (2014). Panorama SIG Libre 2014/Clientes http://wiki.osgeo.org/ wiki/Panorama_SIG_Libre_2014/Clientes\#cite_note-2_[Consulta: 21/07/2017]

OSGeo (2016). Estándares del Open GeoSPatial Consortium http://live.osgeo.org/es/standards/standards.html [Consulta: 21/07/2017]

Quesada-Román, A. (2015). La Mapoteca Virtual de la Universidad Nacional de Costa Rica. Perspectivas, (11), 13. www.revistas.una.ac.cr/index.php/perspectivas/article/ view/ 7530 [Consulta: 21/07/2017].

-Ramos, N.; Roset, R. (2012). Georreferenciación de Mapas antiguos con la ayuda de usuarios. Revista Catalana de Geografía, (XVII), 46. www.rcg.cat/articles.php?id=257 [Consulta: 21/07/2017]

Universidad Nacional de Costa Rica (2017). "Universidad Nacional de Costa Rica. Mapoteca Virtual”. http://www.repositorio.una.ac.cr/handle/11056/7075 [Consulta: 21/07/2017]

Witmer, A.; Hagan, J.; Scaffidi, B.; Hancock, J. (2006). Automated georeferencing of digitized map images. https://www.google.com/patents/US20060041375 [Consulta: 21/07/2017] 